Pacific

Journal of

Mathematics

\title{
CONSTANT MEAN CURVATURE GRAPHS
} IN A STRIP OF $\mathbb{R}^{2}$

RAFAEL LÓPEZ 


\title{
CONSTANT MEAN CURVATURE GRAPHS IN A STRIP OF $\mathbb{R}^{2}$
}

\author{
RAFAEL LÓPEZ
}

\begin{abstract}
We consider the Dirichlet problem for the constant mean curvature surface equation on domains of an infinite strip of the plane. We give sufficient conditions for the existence of smooth solutions provided that the boundary satisfies a certain exterior circle condition. A feature of the work is the use of pieces of nodoids as barriers to make $C^{0}$ and $C^{1}$ a priori estimates respectively.
\end{abstract}

\section{Introduction.}

Let $\Omega$ be a smooth domain in $\mathbb{R}^{2}$. We consider the classical Dirichlet problem of the constant mean curvature equation:

$$
\begin{aligned}
& E(u) \equiv \operatorname{div} \frac{\nabla u}{\sqrt{1+|\nabla u|^{2}}}=-2 H \quad \text { on } \Omega \\
& u=\phi \quad \text { along } \partial \Omega,
\end{aligned}
$$

where $H$ is a given nonzero number and $\phi$ is a smooth function on $\partial \Omega$. The graph of a solution $u \in C^{2}(\Omega) \cap C^{0}(\bar{\Omega})$ is a surface with constant mean curvature $H$ spanning the space curve given by the graph of $\phi$. The orientation of this graph is given by $N=(\nabla u,-1) / \sqrt{1+|\nabla u|^{2}}$, that is, $N$ points downward. From the physical viewpoint, a soap film in equilibrium between two regions of different gas pressure - no gravity - is modeled mathematically by a surface with nonzero constant mean curvature and this constant $H$ represents the pressure difference across the soap film.

The general result of existence of solutions for (1)-(2) is done by Serrin in [Se] when $\Omega$ is a bounded convex domain:

Serrin's Theorem. Let $H>0$. Let $\Omega$ be a bounded smooth domain of $\mathbb{R}^{2}$ such that the curvature $\kappa$ of $\partial \Omega$ with respect to the inner orientation satisfies $\kappa \geq 2 H$. Then given a continuous function $\phi$ on $\partial \Omega$, there exists a unique solution of (1)-(2).

We mention that some results of existence with zero Dirichlet data on the boundary of a bounded convex domain have been obtained in [Lo1, Lo2, Lo3, LM, Mo, PP]. In the most part of them, known surfaces of constant mean curvature, such as spherical caps or cylinders, are used as barriers for 
obtaining the $C^{1}$ estimates in the Leray-Schauder approach. The reason is due that these surfaces fit well with the convexity of the domain $\Omega$.

A characteristic feature of the present article is the use of a family of rotationally symmetric surfaces with constant mean curvature, exactly pieces of nodoids, as barriers to make $C^{0}$ and $C^{1}$ estimates respectively. We use these surfaces in a particular sense introduced by Finn $[\mathbf{F i 2}]$ in his studies of capillary surfaces. See also the subsequent papers [Fi3, Fi4, Fi5].

When $\Omega$ is an infinite strip, Finn proved that the solvability of (1) in $\Omega$ implies that the width $w$ of the strip satisfies $w \leq 1 /|H|$. Finn [Fi1] conjectured that the half-cylinder of radius $1 /(2|H|)$ is the only graph with constant mean curvature $H$ in a strip of width $1 /|H|$. Wang [Wa] and Collin $[\mathbf{C o}]$ independently showed that other different solutions exist, so Finn's conjecture is not true.

Recently, the present author has proved that if $\Omega$ is an unbounded convex domain, the necessary and sufficient condition in order to have solutions of (1) with zero boundary data is that $\Omega$ is included in a strip of width $1 /|H|$ [Lo2]. Finally, we mention that in the minimal case $(H=0)$, the study of the Dirichlet problem, as well existence as uniqueness, has been extensively studied in the literature.

The work is organized as follows. In Section 2 we state our main results. Section 3 describes a family of nodoids and its main properties that will be used in our proofs. In the remaining sections we show the theorems stated in Section 2. Sections 4 and 5 are devoted to show results of existence on unbounded domains included in strips of the plane, whereas Section 6 proves an existence theorem for bounded domains.

With respect to the techniques employed, we refer to the books Courant \& Hilbert $[\mathbf{C H}]$ and Gilbarg \& Trudinger $[\mathbf{G T}]$ for general guides of the quasilinear elliptic equations theory and that we will use as references in our proofs. In particular, we refer to the Perron's method, $C^{0}$ and $C^{1}$ estimates and the Leray-Shauder theory.

\section{Statement of results.}

Consider $\Omega$ an infinite strip of width $w$ given by

$$
\Omega=\left\{(x, y) \in \mathbb{R}^{2} ; 0<y<w\right\} .
$$

Given a smooth function $f: \mathbb{R} \rightarrow \mathbb{R}$, we associate the function $\phi_{f}$ defined on $\partial \Omega$ by

$$
\phi_{f}(x, 0)=\phi_{f}(x, w)=f(x) .
$$

A solution $u$ of (1) with $\phi_{f}$ as boundary data is a graph on $\Omega$ spanning the curve (with two components)

$$
\Gamma(f)=\{(x, 0, f(x)) ; x \in \mathbb{R}\} \cup\{(x, w, f(x)) ; x \in \mathbb{R}\} .
$$


Let $\Omega$ be a smooth domain in $\mathbb{R}^{2}$. Recall that $\Omega$ satisfies a uniform exterior $R$-circle condition if at each point $p \in \partial \Omega$, there exists a disc $D$ depending on $p$ and with radius $R$, such that $\bar{D} \cap \bar{\Omega}=p$ [GT]. This is equivalent that the curvature of $\partial \Omega$ at $p$, measured with the outward orientation, is smaller than $1 / R$. From the geometric viewpoint, the uniform exterior $R$ circle condition means that a circle $C_{R}$ of radius $R$ can 'roll' outside $\Omega$ along $\partial \Omega$ touching each point of $\partial \Omega$ along its displacement.

Let $f$ be a smooth function defined on $\mathbb{R}$. We say that $f$ satisfies a uniform exterior $R$-circle condition if the same holds for some one of the two domains of $\mathbb{R}^{2} \backslash$ graph $(f)$. By the smoothness of $f$, this condition means that $f$ is $C^{2}$ uniformly bounded by a constant that depends on $R$.

We now establish two theorems for the solvability of the Dirichlet problem (1)-(2). With the notations of Proposition 1 in Section 3 below, our first result concerns to the existence of graphs on infinite strips:

Theorem 1. Let $H>0$ and let $f: \mathbb{R} \rightarrow \mathbb{R}$ be a smooth function that satisfies a uniform exterior $\rho_{R}(H)$-circle condition, $0<R(H)<\infty$. Let $\Omega$ be a strip of width $w=2 h_{R}(H)$. Then there exists a solution of (1)-(2) with $\phi=\phi_{f}$. Moreover u satisfies

$$
f(x)-\frac{1}{2 H} \leq u(x, y) \leq f(x)+\frac{1}{2 H} \quad(x, y) \in \Omega .
$$

The second theorem considers the case $\phi \equiv 0$ in the boundary condition.

Theorem 2. Let $H>0$ and let $\Omega$ be a domain that satisfies a uniform exterior $R(H)$-circle condition, $0<R(H)<\infty$. If $\Omega$ is included in a strip of width $w<2 h_{R}(H)$, then (1) has a unique solution with zero boundary data.

Incidentally, Theorem 2 applies as well unbounded as bounded domains. In particular, $\Omega$ can be a planar domain of finite type, as for example, an annulus. In order to show the techniques employed, we shall distinguish both cases. The preceding theorem can be extended to more general situations in terms of the size of the domain $\Omega$ (see Theorem 4 in Section 6).

\section{The 1-parameter family of nodoids.}

Delaunay surfaces in Euclidean space are the surfaces of revolution that are obtained by tracing a focus of a conic section which is rolled without slipping along a line and revolving the resulting curve around that line. When the conic is a ellipse, the surface is an unduloid and if the conic is a hyperbola, the surface is a nodoid. Both families of surfaces are the only surfaces of revolution and with nonzero constant mean curvature in Euclidean space [De] (cylinders and spheres are critical cases of undulois). In addition, for each mean curvature, a deformation allows to parametrize by a real parameter all Delaunay surfaces with the same mean curvature. Exactly, 
if $H$ is a fix number, the deformation starts with the cylinder of radius $1 /(2|H|)$. Next, the surfaces transform into unduloids until that we come to a stack of tangent spheres of radius $1 /|H|$. From here, the deformation gives nodoids bigger and bigger.

Our interest lies in the role that nodoids play. In contrast to unduloids, nodoids have self-intersections. The key property that we shall use of nodoids is the following. Fix $H>0$. A nodoid with mean curvature $H$ contains a piece catenoid-shaped with mean curvature vector pointing outward and that allows to use it as a barrier to compare with surfaces with the same mean curvature. See Figure 1. In addition, in the uniparametric family of nodois with mean curvature $H$, these pieces can be bigger and bigger in a sense that it will be precised later.

Recall the construction of nodoids and some of their properties that we will use later. Let $\gamma(u)=(r(u), 0, u), u \in I$, be a smooth curve defined on an open interval $I$ of real numbers including zero and $r>0$. Applying a rotational motion with respect to the $z$-axis on $\gamma$, we define the surface of revolution

$$
\mathbf{X}(u, \theta)=(r(u) \cos \theta, r(u) \sin \theta, u) .
$$

Choose the orientation $N(u, \theta)=\left(\cos \theta, \sin \theta,-r^{\prime}\right) / \sqrt{1+r^{\prime 2}}$ and assume that the mean curvature $H$ is constant. Let us remark that $\langle N(u, \theta), \mathbf{X}(u, \theta)\rangle$ $>0$. The mean curvature is given by the nonlinear second order differential equation

$$
2 H=-\frac{1}{r\left(1+r^{\prime 2}\right)^{1 / 2}}+\frac{r^{\prime \prime}}{\left(1+r^{\prime 2}\right)^{3 / 2}},
$$

where the principal curvatures are each one of the two summands. With the chosen orientation, the mean curvature $H$ is positive.

Multiplying in (4) by $r r^{\prime}$, we derive a first integral of this equation: There exists a constant $c \in \mathbb{R}$ such that

$$
H r^{2}=-\frac{r}{\sqrt{1+r^{\prime 2}}}+c
$$

(Useful bibliography on Delaunay surfaces is [Ee] and [Ka, Appendix A].) Initial conditions on (5) make that the surface constructed by rotating $\gamma$ is an unduloid or a nodoid.

We focus in the latter case: There exist $h, \rho>0$, where $r:[-h, h] \rightarrow$ $[-\rho, \rho]$ is an even function and the initial condition $r(0)=t>0$ is the minimum value of $r$. Moreover, $r(h)=\rho$ and $r^{\prime}(h)=+\infty$.

Since $r^{\prime}(0)=0, H t^{2}+t=c$. Then $H \rho^{2}=c$. From (5),

$$
t=\frac{-1+\sqrt{1+4 H c}}{2 H} \text {. }
$$




$$
\rho=\sqrt{\frac{H t^{2}+t}{H}}=\sqrt{\frac{c}{H}} .
$$

When convenient, we indicate the dependence of the parameter $t$ by a subscript $t$ and by $(H)$ the dependence of $H$. Write $\mathcal{N}$ the nodoid obtained by rotating $\gamma$ and $\gamma$ defined on the interval $[-h, h]$.

Remark that $\rho_{t}(H)-t(H)$ is increasing in $t$ and

$$
\rho_{t}(H)-t(H)=\frac{1}{2 H}\left(1-\frac{1}{2 \sqrt{H c}+\sqrt{1+4 H c}}\right) \rightarrow \frac{1}{2 H},
$$

as $t \rightarrow \infty$. Change coordinates and let us view $\gamma$ as a graph on the $x$-axis. Then the surface is given by

$$
\mathbf{X}(x, \theta)=(x \cos \theta, x \sin \theta, z(x)) .
$$

Now the mean curvature $H$ is

$$
H x^{2}=\frac{x z^{\prime}}{\sqrt{1+z^{\prime 2}}}+c,
$$

and

$$
z(t)=0, z^{\prime}(t)=+\infty
$$

Therefore we have

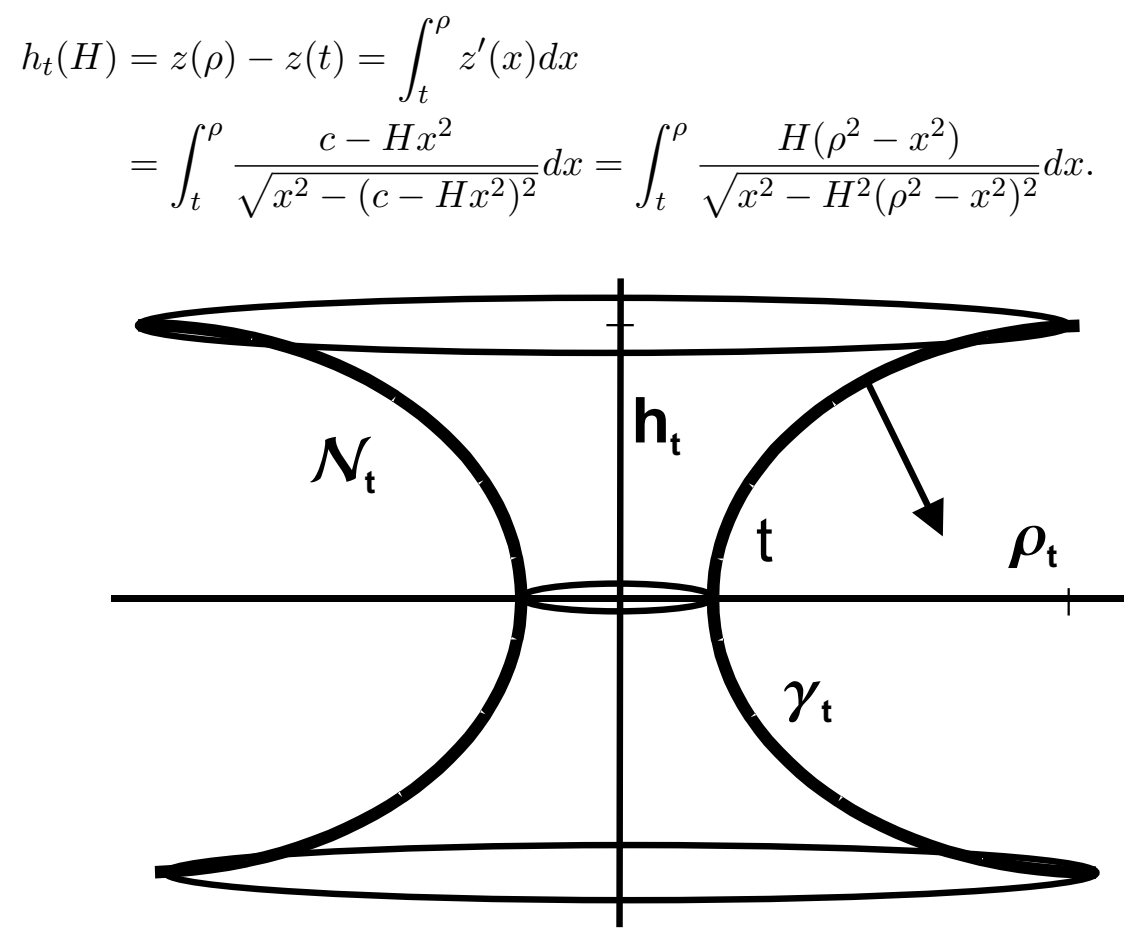

Figure 1. A uniparametric family of nodoids. 
With the preceding notations, we summarize these properties:

Proposition 1. Let $H>0$. There exists a uniparametric family of nodoids $\left\{\mathcal{N}_{t} ; t>0\right\}$ with constant mean curvature $H$ given by the rotation of a curve $\gamma_{t}$ around the $z$-axis and with the following properties (see Figure 1):

(a) The curve $\gamma_{t}$ is a graph on the interval $\left(-h_{t}, h_{t}\right)$, and symmetric with respect to the origin.

(b) The curve $\gamma_{t}$ is defined in the interval $\left[-h_{t}, h_{t}\right]$ and with horizontal tangent at $\pm h_{t}$. Thus $\mathcal{N}_{t}$ is included in the slab $S_{t}:|z|<h_{t}$ and is tangent to it.

(c) The mean curvature vector points outside the bounded domain $W_{t}$ determined by $\mathcal{N}_{t}$ and the slab $S_{t}$.

(d) The circle $C_{t}$ of $\mathcal{N}_{t}$ with the smallest radius is given by $x^{2}+y^{2}=t^{2}$, $z=0$.

(e) The function $h_{t}(H)$ is strictly increasing on $t$ and

$$
\lim _{t \rightarrow 0} h_{t}(H)=0 \quad \lim _{t \rightarrow \infty} h_{t}(H)=\frac{1}{2 H} .
$$

(f) The function $\rho_{t}(H)$ is strictly increasing on $t$ and

$$
\lim _{t \rightarrow 0} \rho_{t}(H)=0 \quad \lim _{t \rightarrow \infty} \rho_{t}(H)=\infty \quad \lim _{t \rightarrow \infty} \rho_{t}(H)-t(H)=\frac{1}{2 H} .
$$

\section{Proof of Theorem 1.}

This section is devoted to the existence of (1)-(2), $\phi=\phi_{f}$, on an infinite strip. Without loss of generality, let

$$
\Omega=\left\{(x, y) \in \mathbb{R}^{2} ; 0<y<w\right\} .
$$

Firstly, we need a theorem of existence of minimal graphs on a strip. The following result is a consequence of an existence theorem due to Nitsche [Ni] and Jenkins and Serrin [JS] for bounded domains with piecewise smooth convex boundary (see also $[\mathbf{E R}]$ ).

Proposition 2. Let $\Omega$ be a strip and let $f$ be a continuous function on $\partial \Omega$. Then there exists a solution $\varphi$ of the Dirichlet problem

$$
\begin{aligned}
& \operatorname{div} \frac{\nabla u}{\sqrt{1+|\nabla u|^{2}}}=0 \quad \text { on } \Omega \\
& u=f \quad \text { along } \partial \Omega .
\end{aligned}
$$

Proof. For each $n \in \mathbb{Z}$ and $n>w$, consider the points $P_{n}=(n, 0), Q_{n}=$ $(n, w)$, and the intervals $I_{n}=\{n\} \times(0, w)$. Construct an exhaustion $\Omega_{n} \subset$ $\Omega_{n+1}$ of $\Omega$, where $\Omega_{n}$ is the rectangular domain bounded by the segments $I_{-n} \cup I_{n} \cup\left(P_{-n}, P_{n}\right) \cup\left(Q_{-n}, Q_{n}\right)$. Since the line segments of its boundary satisfy

length $\left(I_{-n}\right)+$ length $\left(I_{n}\right)<$ length $\left(P_{-n}, P_{n}\right)+$ length $\left(Q_{-n}, Q_{n}\right)$, 
the mentioned Nitsche-Jenkins-Serrin's theorem shows the existence of a sequence $v_{n}$ (resp. $v_{n}^{\prime}$ ) of solutions of the minimal surface equation on $\Omega_{n}$ assuming the value $f$ along $\left(P_{-n}, P_{n}\right) \cup\left(Q_{-n}, Q_{n}\right)$ and $+\infty$ (resp. $-\infty$ ) along $I_{-n} \cup I_{n}$. Then $v_{n}$ (resp. $v_{n}^{\prime}$ ) is a decreasing (resp. increasing) sequence. If $C \subset \Omega$ is a compact set and $C \subset \Omega_{m}$, then for $n \geq m$, we have on $C$ the inequalities:

$$
\inf _{C} v_{m}^{\prime} \leq v_{m}^{\prime} \leq v_{n}^{\prime} \leq v_{n} \leq v_{m} \leq \sup _{C} v_{m} .
$$

A compactness theorem, together with a diagonal argument shows that $v_{n}$ converges to a minimal solution $\varphi$ on $\Omega$. By comparing with Scherk's surface, we obtain $\varphi=f$ along $\partial \Omega$, as desired.

Without loss of generality we assume that the domain of $\mathbb{R}^{2} \backslash$ graph $(f)$ that satisfies the uniform exterior $\rho_{R}$-circle condition lies above the graph $(f)$. Let $\mathcal{N}_{R}$ be the nodoid of constant mean curvature $H$ given by Proposition 1 for the parameter $t=R$. This surface is bounded by two parallel, coaxial circles of radii $\rho_{R}$, at distance $w=2 h_{R}$ apart.

First, assuming existence, we prove inequality (3). Let $u$ be a solution of (1)-(2) and let $M$ be its graph. Place now $\mathcal{N}_{R}$ above $\Omega$ and the graph of $f$ and so that the axis of $\mathcal{N}_{R}$ is parallel to the $y$-axis, and the boundary curves of $\mathcal{N}_{R}$ are in the planes $y=0, y=w$ respectively. Place $\mathcal{N}_{R}$ above $M$ so that $\mathcal{N}_{R} \cap M=\emptyset$. Move $\mathcal{N}_{R}$ vertically downward until to touch the first time with $M$. By the maximum principle and since $M$ is a graph, the first contact with $M$ can not be at an interior point of $M$ because the mean curvature vector of $\mathcal{N}_{R}$ points down (see Proposition 1 (c)). Therefore no accident will occur before reaching $\Gamma(f)$. This implies that the first point of contact occurs when $\partial \mathcal{N}_{R}$ touches $\Gamma(f)$. See Figure 2 .

Now one can move $\mathcal{N}_{R}$ in the $x$-direction, so that $\partial \mathcal{N}_{R}$ rolls along $\Gamma(f)$, with exactly one point of contact each time, and the axis of $\mathcal{N}_{R}$ remains parallel to the $y$-axis. Recall that the mean curvature vector of $\mathcal{N}_{R}$ points outside of $W_{R}$ (Proposition 1 (c)). Then the uniform exterior $\rho_{R}$-circle condition of $f$ and the maximum principle assure that in this displacement, $\mathcal{N}_{R}$ touches $M$ only at boundary point of $M$ and in each one of them. The same argument holds if we consider $\mathcal{N}_{R}$ below $M$ and we ascend until to contact with $M$, and rolling next. This concludes the proof of the estimate (3).

We now begin with the proof of the part of existence in Theorem 1 . The proof is by means of the Perron process $([\mathbf{C H}]$, pp. 306-312). The reader is referred to [Lo2] for an example in the same context. For this, one needs to assure that we can solve in the small; i.e., provided that for small enough discs there exist solutions with arbitrary continuous boundary values.

Let $v$ be a continuous function on $\bar{\Omega}$ and let $D$ denote a closed disc lying in $\Omega$. Let $M_{D}(v)$ denote the continuous function which coincides with $v$ in $\Omega \backslash D$ and which is a solution of the Dirichlet problem (1)-(2) in $D$ with 


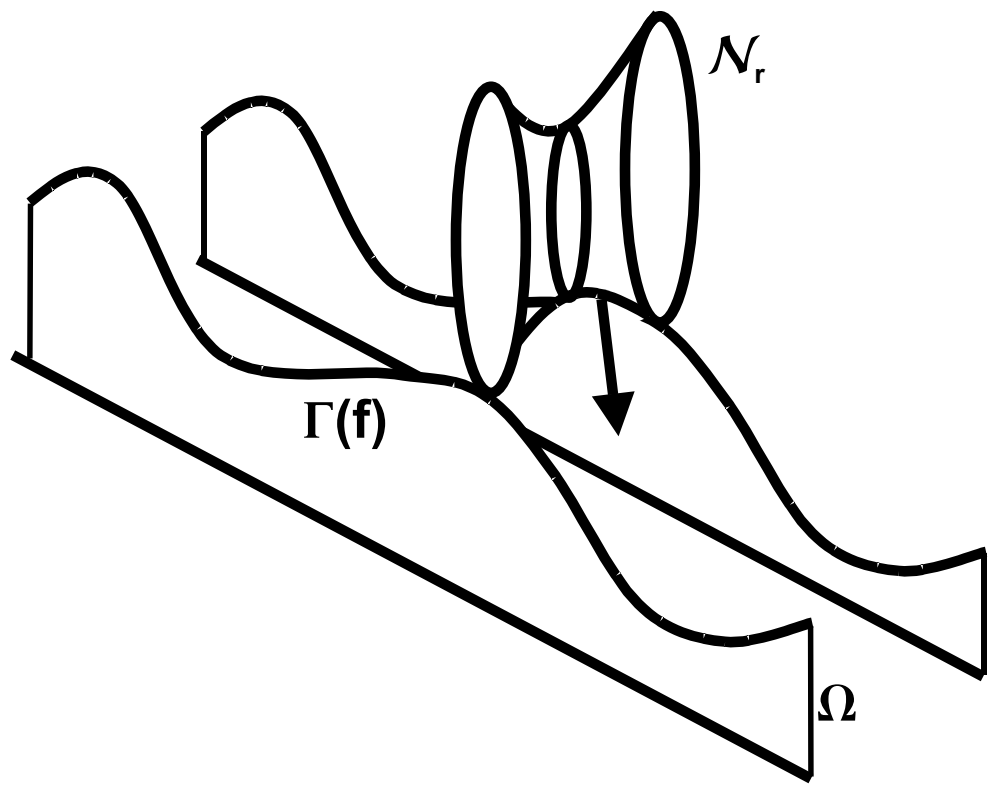

Figure 2. Proof of Theorem 1.

$\phi=v_{\mid \partial D}$. The existence of $M_{D}(v)$ is assured by Serrin's theorem, since the radius of $D$ is less than $1 /(2 H)$.

The function $v$ is said to be a sub-solution in $\Omega$ if

$$
v \leq M_{D}(v)
$$

for every closed disc $D$ in $\Omega$. Subsolutions have the following two properties:

Proposition 3. With the preceding notations and for every disc $D$ in $\Omega$, we have:

1. $M_{D}$ is increasing.

2. If $v$ is a subsolution, then $M_{D}(v)$ does.

Proof.

1. This is an immediate consequence of the maximum principle.

2. Let $D^{\prime}$ be a disc in $\Omega$. We have to prove that $M_{D}(v) \leq M_{D^{\prime}}\left(M_{D}(v)\right)$ for each subsolution $v$. It suffices to show the inequality

$$
M_{D}(v) \leq M_{D^{\prime}}\left(M_{D}(v)\right)
$$

in $D^{\prime}$. Since $M_{D}$ is increasing and $v$ is a subsolution, we have $v \leq$ $M_{D^{\prime}}(v) \leq M_{D^{\prime}}\left(M_{D}(v)\right)$. On $D^{\prime} \backslash D, v=M_{D}(v)$ and the inequality (8) holds in $D^{\prime} \backslash D$. On the other hand, $M_{D}(v) \leq M_{D^{\prime}}\left(M_{D}(v)\right)$ along the boundary $\partial\left(D \cap D^{\prime}\right)$, and the maximum principle assures that the same inequality holds in $D^{\prime} \cap D$, obtaining the desired inequality (8). 
Let $\Omega_{R}$ be the region in $\Omega$ to which $\mathcal{N}_{R}$ projects by vertical projection. Let $p \in \partial \Omega$, that is, $p=\left(x_{0}, \epsilon\right)$, where $\epsilon=0$ or $\epsilon=w$. Place $\mathcal{N}_{R}$ so that the one of the two points at lowest height (necessarily boundary points) project on $p$. Consider the half part of $\mathcal{N}_{R}$ that lies below the horizontal plane containing its axis. We call $\mathcal{N}_{R}^{*}$ this piece of $\mathcal{N}_{R}$. Then $\mathcal{N}_{R}^{*}$ is a graph on $\Omega_{R}$ with mean curvature $H$ and with the unit normal vector field pointing downward. Put $\mathcal{N}_{R}^{*}$ so that the point $\left(p, f\left(x_{0}\right)\right)$ belong to $\partial \mathcal{N}_{R}^{*}$. Denote by $\nu_{p}$ the function on $\Omega_{R}$ whose graph is $\mathcal{N}_{R}^{*}$ and also denote $\Omega_{p}=\Omega_{R}$ to indicate the dependence of $p$. Finally, let $\varphi$ be the minimal solution given by Proposition 2 .

Let $\Sigma$ be the family of function $\nu_{p}$ with $p \in \partial \Omega$. On the one hand, we have $E\left(\nu_{p}\right)=-2 H$ and $f \leq \nu_{p}$ along $\partial \Omega_{R} \cap \partial \Omega$, on the other, $E(\varphi)=$ $0>-2 H=E\left(\nu_{p}\right)$. By the maximum principle, $\varphi \leq M_{D}(\varphi)$ for each disc in $\Omega$ and then, $\varphi$ is a subsolution that satisfies $\varphi \leq \nu_{p}$, for each $\nu_{p} \in \Sigma$. Introduce the concept of subfunction relative to $f$. If $v$ is a subsolution in $\Omega$ and $v \leq f$ along $\partial \Omega$, then $v$ is said to be a subfunction relative to $f$. We are going to prove that between the function $\nu_{p}$ of $\Sigma$ and the subsolution $\varphi$, there exists a solution of (1). Let $F_{f}$ be the class of all subfunctions $v$ such that $\varphi \leq v \leq \nu_{p}$ in $\Omega_{p}$, for each $p \in \partial \Omega$.

The functions of $F_{f}$ are uniformly bounded in every compact set of $\bar{\Omega}$. Since $\varphi \in F_{f}$, we can define for each $(x, y) \in \bar{\Omega}$,

$$
u(x, y)=\sup _{v \in F_{f}} v(x, y) .
$$

By virtue of Proposition 3 , the set $F_{f}$ is stable by $M_{D}$, i.e., if $v \in F_{f}$, then $M_{D}(v) \in F_{f}$. Thus

$$
u=\sup _{v \in F_{f}} M_{D}(v)(x, y) .
$$

Using the compactness principle and the maximum principle, it now follows exactly as in $[\mathbf{C H}]$ that $u$ is a solution of Equation (1).

Finally, a standard barrier argument may now be employed to prove that $u$ is continuous in $\bar{\Omega}$ and assumes the boundary value $\phi_{f}$ at each point of the boundary for which a barrier can be constructed. The fact that $u$ is continuous in $\bar{\Omega}$ is a consequence of Harnack's principle (see [Se]). In order to show that $u$ takes the value $\phi$ on $\partial \Omega$ we use the functions $\nu_{p}$ as local barriers. Indeed, let $p \in \partial \Omega$. Then the functions $\nu_{p}$ and $\varphi$ are a modulus of continuity in a neighbourhood of $p$. Since $\nu_{p}(p)=\varphi(p)=f(x)$, with $p=(x, \epsilon)$, we obtain $u(p)=f(x)$ and this completes the proof of Theorem 1.

Example 1. We illustrate Theorem 1 with an example. Let $H=1$ and $f(x)=\sin x$. Then $f$ satisfies a uniform exterior 1-circle condition. Here $\rho_{R}=1$ and by $(6)-(7), R=(\sqrt{5}-1) / 2$. Then Theorem 1 assures the 
existence of a graph on a strip of width $w=2 h_{R}$ of constant mean curvature $H=1$ and spanning $\Gamma(f)$. Here

$$
h_{R}=\int_{R}^{1} \frac{1-x^{2}}{\sqrt{x^{2}-\left(1-x^{2}\right)^{2}}} d x \approx 0.3196
$$

and the width of the strip is $\approx 0.6392$.

Remark 1. Theorem 1 is a generalization of the result derived by Collin in [Co]. Collin proved the existence of solutions of (1)-(2) in a strip of width $1 /|H|$ for the boundary value $\phi_{f}$ and $f$ is a convex function. Indeed, if $f$ is a convex function, $f$ satisfies a uniform exterior $\rho_{R}$-circle condition for the critical case $\rho_{R}=+\infty$. Then $h_{\infty}=1 /(2|H|)$ (Proposition $1(\mathrm{e})$ ) and Theorem 1 assures the existence of a strip of width $2 h_{\infty}=1 /|H|$.

Remark 2. Indeed the arguments described in the proof prove that Theorem 1 holds for strips of width less than $2 h_{R}$.

\section{Proof of Theorem 2 for unbounded domains.}

As we announced in Section 2, we make a difference in Theorem 2 between the unbounded case and the bounded one. This section is devoted to the first situation. More precisely, the following result holds:

Theorem 3. Let $H>0$ and let $\Omega$ be an unbounded domain that satisfies a uniform exterior $R(H)$-circle condition, $0<R(H)<\infty$. If $\Omega$ is included in a strip of width $w<2 h_{R}(H)$, then (1) has a unique solution with zero boundary data.

Proof. Consider $\Omega \subset\left\{(x, y) \in \mathbb{R}^{2} ; 0<y<w\right\}$. Solutions of (1) with zero boundary data are bounded [Me, Lemma 2.4]. Consider $\mathcal{N}_{R}$ as in proof of Theorem 1 and let $u$ be a solution of (1)-(2) for $\phi \equiv 0$. Without loss of generality, we assume that $u \geq 0$. The same argument that is done in proving the height estimate holds now to find a constant $C$ depending only on $\partial \Omega$, such that $0 \leq u<C<h_{R}$ (notice that the width $w$ of the strip is strictly less than $2 h_{R}$ ).

Let us fix $H$. Consider the corresponding family of nodoids $\mathcal{N}_{t}$. Let $r_{1}<R$ so that $h_{r_{1}}=C$. Place $\mathcal{N}_{r_{1}}$ so that the axis of $\mathcal{N}_{r_{1}}$ is the $z$-axis. Consider $\mathcal{N}^{r_{1}}$ the piece of $\mathcal{N}_{r_{1}}$ obtained by rotation with respect to the $z$-axis the curve $\gamma_{r_{1}}$ defined in the interval $\left[0, h_{r_{1}}\right]$ of the $z$-axis.

We move down $\mathcal{N}^{r_{1}}$ a small vertical displacement such that the new radius $r$ of the circle intersection with the horizontal plane $P: z=0$ satisfies $r_{1}<r<R$ (and then $C<h_{r}$ ). Denote again by $\mathcal{N}^{r}$ this part above the plane $P$.

Denote $\Omega^{r}$ the annulus in $\Omega$ to which $\mathcal{N}^{r}$ projects by vertical projections. The radii of $\Omega^{r}$ are $r$ and $\rho_{r}, r<\rho_{r}$, where the first circle lies in the plane $P$ 
and the second one at height $h_{r}$. Denote $\mu$ the function on $\Omega^{r}$ whose graph is $\mathcal{N}^{r}$.

Arguing as in the proof of Theorem 1, introduce the class of subfunctions relative to the zero function. Now, we have the minimal solution $\varphi=0$ on $\Omega$. Consider $p \in \partial \Omega$ and place $\mathcal{N}^{r}$ such that the disk $D_{r}$ defined by the circle $\partial \mathcal{N}^{r} \cap P$ of radius $r$ satisfies $D_{r} \subset \mathbb{R}^{2} \backslash \Omega$ and $\overline{D_{r}} \cap \bar{\Omega}=p$. This is possible because $\Omega$ satisfies a uniform exterior $R$-circle condition and $r<R$. Denote $\mu_{r}=\mu_{p}$ and $\Omega^{r}=\Omega^{p}$ to indicate the dependence on $p$.

Let $\Sigma$ be the family of functions $\mu_{p}$ with $p \in \partial \Omega$. Then we have again that $0 \leq \mu_{p}$ on $\Omega^{p} \cap \Omega$. Now the steps to follow are analogous. Let $F$ be the class of all subfunctions $v$ such that $0 \leq v \leq \mu_{p}$ in $\Omega^{p} \cap \Omega$ for each $p \in \partial \Omega$. Recall $M$ lies above the plane $P$. This set is not empty since $0 \in F$ and

$$
u(x, y)=\sup _{v \in F} v(x, y)=\sup _{v \in F} M_{D}(v)(x, y)
$$

is a solution of (1) in $\Omega$ and continuous up to $\bar{\Omega}$.

We use the functions $\mu_{p}$ and 0 in a neighbourhood of $p$ for the modulus of continuity. This occurs as follows: We ascend $\mathcal{N}^{r}$ until does not touch the graph $M$ of $u$. Then we descend it. The maximum principle assures the nonexistence of interior contact points between $\mathcal{N}^{r}$ and $M$ when $\mathcal{N}^{r}$ arrives to the plane $P$. See Figure 3. Let us remark that the height of $M$ is strictly less than $C$ and $C<h_{r}$, with $h_{r}$ the height of $\mathcal{N}^{r}$ and that the mean curvature vector of $\mathcal{N}^{r}$ points down. Finally, we use the fact that $\mu_{p}(p)=0=\varphi(p)$.

The uniqueness is a result obtained by Miklyukov [Mi], Hwang $[\mathbf{H w}]$ and Collin and Krust $[\mathbf{C K}]$ independently. This concludes the proof of Theorem 3.

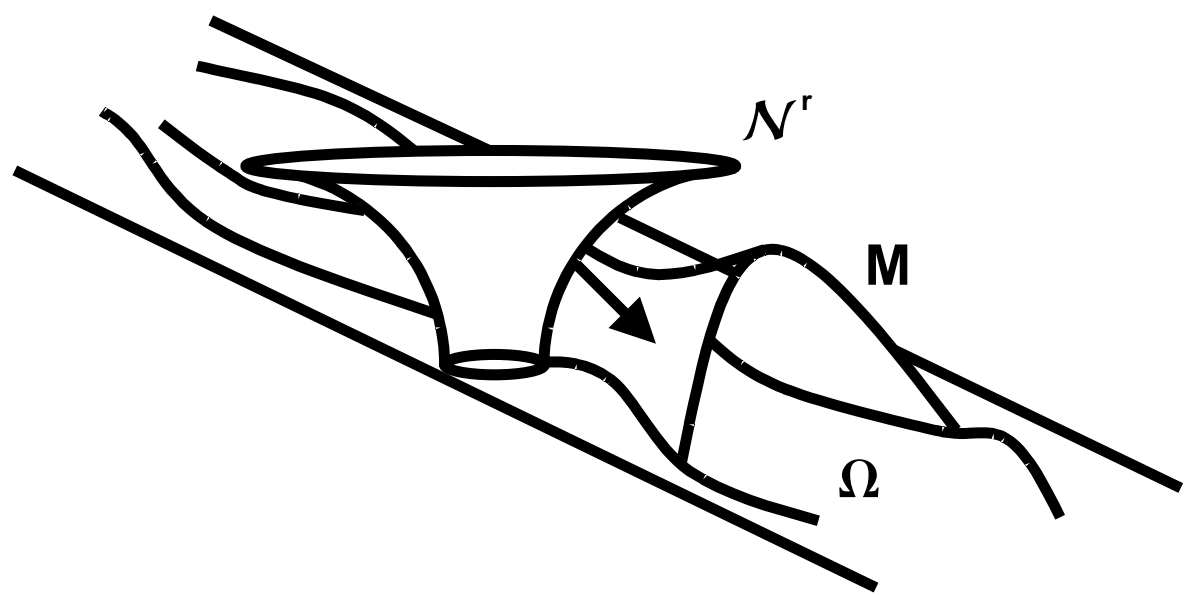

Figure 3. Proof of Theorem 3. 
Example 2. Let $H=0.1$. Consider the functions $f(x)=\sin x$ and $g(x)=$ $0.5+f(x)$. Let $\Omega$ be the domain of the $(x, y)$-plane bounded by the graphs of $f$ and $g$. Then $\Omega$ satisfies a uniform exterior 1-circle condition. Now (6)-(7) imply $R=1$ and $\rho_{1}=\sqrt{11}$. Here

$$
h_{1}=\int_{1}^{\sqrt{11}} \frac{0.1\left(11-x^{2}\right)}{\sqrt{x^{2}-0.01\left(11-x^{2}\right)^{2}}} d x \approx 1.3201 .
$$

Since $\Omega$ is included in a strip of width $2.5<2 h_{1}$, Theorem 3 assures the existence of a solution of (1)-(2) on $\Omega$ with $H=0.1$ and $\phi=0$.

\section{An existence theorem for bounded domains.}

In this section, we shall prove the existence of graphs with constant mean curvature on bounded planar domains provided that the height of the corresponding graph is small enough so that we can compare with pieces of nodoids as in Theorems 1 and 3. Since the same argument applies for other situations, we restate Theorem 2 in a more general version.

Theorem 4. Let $H>0$ and let $\Omega$ be a bounded region that satisfies a uniform exterior $R$-circle condition, $0<R<\infty$. In any of the following cases, there exists a solution of (1) for zero boundary data:

1. The domain $\Omega$ is included in a strip of width $2 h_{R}$.

2. The length $L$ of $\partial \Omega$ satisfies $L H<2 \pi \sqrt{H h_{R}\left(2-H h_{R}\right)}$.

3. The area $a(\Omega)$ of $\Omega$ satisfies

$$
a(\Omega)<\frac{2 \pi h_{R}}{H\left(1+2 H h_{R}\right)} .
$$

4. The domain $\Omega$ is included in a circular disc of radius $\sqrt{\left(2-H h_{R}\right) h_{R} / H}$. Proof. First we claim that any hypothesis in Theorem 4 implies that the height of a solution $u$ of (1)-(2), $\phi \equiv 0$, is strictly less than $h_{R}$. Call $P$ the plane $z=0$. Without loss of generality, assume $u \geq 0$ on $\Omega$. Denote $M$ the graph of $u$. Recall that the orientation of $M$ is that points downward. We distinguish the four cases stated in Theorem 4.

1. Without loss of generality, assume that the strip $S$ of width $2 h_{R}$ containing $\Omega$ is $S=\left\{(x, y) \in \mathbb{R}^{2} ; 0<y<2 h_{R}\right\}$. Let $(p, u(p)) \in M$ and consider the nodoids $\mathcal{N}_{R}$ placed as in proof of Theorem 1, that is, the axis of $\mathcal{N}_{R}$ is parallel to the $y$-axis and such that the two coaxial circles of its boundary project on $\partial S$. Also, place $\mathcal{N}_{R}$ so that one of the two endpoints of its axis projects on $p$. Maximum principle as in Theorem 1 concludes that if $\mathcal{N}_{R}$ rests on $S$ along its boundary, the graph $M$ lies between $P$ and $\mathcal{N}_{R}$. Thus we obtain the desired height estimate. 
2. We use the same argument as in [LM, Corollary 4] using the BarbosaDo Carmo isoperimetric inequality. Then the area $A$ of $M$ satisfies $A H /(2 \pi)<h_{R}$ and together with the height bound of Theorem 1 in [LM], we have $u<h_{R}$.

3. Here we use the following height estimate of a graph given in $[$ Lo3 $]$ in terms of the area of $\Omega$ :

$$
h \leq \frac{a(\Omega) H}{2\left(\pi-a(\Omega) H^{2}\right)} .
$$

4. We consider a halfsphere $S$ of radius $1 / H$ in $z \geq 0$ whose boundary lies in $P$ and such that the center of the circle $\partial S$ agrees with the one of the circular disc that contains $\Omega$. Then, translate $S$ upward so $S$ is disjoint from $M$. By the maximum principle, one can translate $S$ downward until that $\partial S$ arrives at $P$. This yields the desired height estimate.

Assuming that the height of prospective solution of the Dirichlet problem is less than $h_{R}$, we study the existence of such graphs. The solvability of the Dirichlet problem can be achieved using the continuity method. Following the usual Leray-Schauder approach (see e.g., [GT, Theorem 13.8]), the Dirichlet problem is solvable if there is $C^{1}(\bar{\Omega})$ a priori estimates for prospective solutions of the Dirichlet problem.

The above reasoning shows the existence of $C^{0}$ a priori estimates. It remains to estimate $|\nabla u|$ on $\Omega$. Standard theory of quasilinear equations assures that it suffices if we obtain a priori bounds of $|\nabla u|$ along $\partial \Omega$. This means that we have to establish estimates of the slope of $M$ along its boundary $\partial M=\partial \Omega$. These estimates will be accomplished with pieces of nodoids as geometrical barrier surfaces and that essentially were done in the proof of Theorem 2.

Following the notation used there, let $\mathcal{N}^{r}$ be the nodoid of constant mean curvature $H$. This surface is a graph on the annulus $\Omega^{r}$. Let $C_{r}=\partial \mathcal{N}^{r} \cap P$ one of the two components of $\partial \mathcal{N}^{r}$. Given $p \in \partial \Omega$, place $\mathcal{N}^{r}$ so that $C_{r} \cap \bar{\Omega}=$ $p$, which is possible for the uniform exterior $R$-circle condition. Then we displace up $\mathcal{N}^{r}$ until does not touch $M$. Descend $\mathcal{N}^{r}$ : Because the height of $M$ is less than $h_{R}$ and $h_{r}$ is the height of $\mathcal{N}^{r}$, the maximum principle guarantees that the first point of contact between $\mathcal{N}^{r}$ and $M$ occurs when $\mathcal{N}^{r}$ arrives at the plane $P$ (see Figure 3 ). In conclusion, the angle of $M$ with the plane $P$ at $p$ does not exceed the angle of $\mathcal{N}^{r}$ at the same point. This gives a uniform bound for the gradient of $u$ along $\partial \Omega$ in terms of $\mathcal{N}^{r}$ and completes the proof.

Remark 3. Convex domains satisfy the uniform exterior $R$-circle condition for $R=+\infty$. In this case, $h_{\infty}=1 /(2 H)$ (Proposition $\left.1(\mathrm{e})\right)$. For this critical value, the correspondent statements of Theorem 4 have been proved. They are respectively: 
1. $\Omega$ is included in a strip of width $1 / H$ [Lo2 $]$.

2. $L H<\sqrt{3} \pi[\mathbf{L M}]$.

3. $a(\Omega) H^{2}<\pi / 2[$ Lo3 $]$.

4. $\Omega$ included in a circular disc of radius $\sqrt{3} /(2 H)$ [Lo1] .

Remark 4. The proof of Theorem 4 assures the existence of the Dirichlet problem (1)-(2), $\phi \equiv 0$, provided we have the uniform exterior $R$-circle condition and some hypothesis that assures us the uniform bound $h_{R}$ for the possible solutions. The above cases in Theorem 4 are examples of it.

Acknowledgements. The author would like to thank the referee for many helpful comments and suggestions.

\section{References}

[Co] P. Collin, Deux exemples de graphes de courbure moyenne constante sur une bande de $\mathbb{R}^{2}$, C.R. Acad. Sci. Paris Sér. I, 311 (1990), 539-542, MR 92i:58041, Zbl 0716.53016.

[CK] P. Collin and R. Krust, Le probléme de Dirichlet pour l'equation des surfaces minimales sur des domains non bornés, Bull. Soc. Math. France, 119 (1991), 443-462, MR 92m:53007, Zbl 0754.53013.

[CH] R. Courant and D. Hilbert, Methods of Mathematical Physics, Vol. II, Interscience, New York, 1962, MR 25 \#4216, Zbl 0099.29504.

[De] C. Delaunay, Sur la surface de révolution dont la courbure moyenne est constante, J. Math. Pure Appl., 6 (1841) 309-320.

[ER] R. Earp and H. Rosenberg, The Dirichlet problem for the minimal surface equation on unbounded planar domains, J. Math. Pures Appl., 68 (1989), 163-183, MR 90m:35072, Zbl 0696.49069.

[Ee] J. Eells, The surfaces of Delaunay, The Mathem. Intell., 9 (1987), 53-57, MR 88h:53011, Zbl 0605.53002.

[Fi1] R. Finn, Remarks relevant to minimal surfaces and to surfaces of prescribed mean curvature, J. d'Anal. Math., 14 (1965), 139-160, MR 32 \#6337, Zbl 0163.34604.

[Fi2] - A limiting geometry for capillary surfaces, Ann. Scuola Norm. Sup. Pisa, 11 (1984), 361-379, MR 87f:49051, Zbl 0564.49022.

[Fi3] The Gauss curvature of an H-graph, Nachr. Akad. Wiss. Göttingen MathPhys. Kl., II(2) (1987), 5-14, MR 88k:53017, Zbl 0645.53003.

[Fi4] Comparison principles in capillarity, in 'Partial Differential Equations and Calculus of Variations', Lectures Notes in Mathematics, 1357, 156-197, SpringerVerlag, Berlin-New York, 1988, MR 90d:53010, Zbl 0692.35006.

[Fi5] Moon surfaces, and boundary behaviour of capillary surfaces for perfect wetting and nonwetting, Proc. London Math. Soc., 57 (1988), 542-576, MR 89m:49076, Zbl 0668.76019.

[GT] D. Gilbarg and N.S. Trudinger, Elliptic Partial Differential Equations of Second Order, Springer-Verlag, Berlin-New York, 1983, MR 86c:35035, Zbl 0562.35001. 
[Hw] J.F. Hwang, Comparison principles and theorems for prescribed mean curvature equation in unbounded domains, Ann. Scuola Norm. Sup. Pisa, 15 (1988), 341-355, MR 90m:35019, Zbl 0705.49022.

[JS] H. Jenkins and J. Serrin, Variational problems of minimal surface type, II. Boundary value problems for the minimal surface equation, Arch. Rat. Mech. Anal., 12 (1963), 185-212, MR 32 \#8221, Zbl 0171.08301.

[Ka] N. Kapouleas, Complete constant mean curvature surfaces in Euclidean three-space, Ann. Math., 131 (1990), 239-330, MR 93a:53007a, Zbl 0699.53007.

[Lo1] R. López, Constant mean curvature surfaces with boundary in Euclidean three-space, Tsukuba J. Math., 23 (1999), 27-36, MR 2000f:53010, Zbl 0980.53013.

[Lo2] - Constant mean curvature graphs on unbounded convex domains, J. Diff. Eq., 171 (2001), 54-62, MR 2002c:53011.

[Lo3] - An existence theorem of constant mean curvature graphs in Euclidean space, to appear in Glasgow Math. J.

[LM] R. López and S. Montiel, Constant mean curvature surfaces with planar boundary, Duke Math. J., 85 (1996), 583-604, MR 97m:53015, Zbl 0877.53008.

[Me] W.H. Meeks III, The topology and geometry of embedded surfaces of constant mean curvature, J. Diff. Geom., 27 (1988), 539-552, MR 89h:53025, Zbl 0617.53007.

[Mi] V.M. Miklyukov, On a new approach to Bernstein's Theorem and related questions for equations of minimal surface type, Mat. Sb., 108(150) (1979), 268-289; English transl. in Math. USSR, 36 (1980), 251-271, MR 80e:53005, Zbl 0488.49029.

[Mo] S. Montiel, A height estimate for $H$-surfaces and existence of $H$-graphs, Amer. J. Math., 123(3) (2001), 505-514, MR 2002c:53012.

[Ni] J.C.C. Nitsche, On new results in the theory of minimal surfaces, Bull. Amer. Math. Soc., 71 (1965), 195-270, MR 30,4200, Zbl 0135.21701.

[PP] L.E. Payne and G.A. Philippin, Some maximum principles for nonlinear elliptic equations in divergence form with applications to capillary surfaces and to surfaces of constant mean curvature, Nonlinear Analysis, Th. Meth. App., 3 (1979), 193-211, MR 80h:35056, Zbl 0408.35015.

[Se] J. Serrin, The problem of Dirichlet for quasilinear elliptic equations with many independent variables, Philos. Trans. Roy. Soc. London Ser. A, 264 (1969), 413496, MR 43 \#7772, Zbl 0181.38003.

[Wa] A.N. Wang, Constant mean curvature surfaces on a strip, Pacific J. Math., 145 (1990), 395-396, MR 91i:53016, Zbl 0724.49026.

Received January 4, 2001. This research was partially supported by DGICYT grant number BFM2001-2967.

Departamento de Geometría y Topología

UNIVERSIDAD DE GRANADA

18071 Granada, Spain

E-mail address: rcamino@goliat.ugr.es 